\title{
The Mitochondrial Permeability Transition Pore and Nitric Oxide Synthase Mediate Early Mitochondrial Depolarization in Astrocytes during Oxygen-Glucose Deprivation
}

\author{
Susan A. Reichert, Jeong Sook Kim-Han, and Laura L. Dugan \\ Department of Neurology and Center for the Study of Nervous System Injury, Washington University School of Medicine, \\ St. Louis, Missouri 63110
}

Recent studies suggest that the degree of mitochondrial dysfunction in cerebral ischemia may be an important determinant of the final extent of tissue injury. Although loss of mitochondrial membrane potential $\left(\psi_{m}\right)$, one index of mitochondrial dysfunction, has been documented in neurons exposed to ischemic conditions, it is not yet known whether astrocytes, which are relatively resistant to ischemic injury, experience changes in $\psi_{m}$ under similar conditions. To address this, we exposed cortical astrocytes cultured alone or with neurons to oxygen-glucose deprivation (OGD) and monitored $\psi_{m}$ using tetramethylrhodamine ethyl ester. Both neurons and astrocytes exhibited profound loss of $\psi_{m}$ after 45-60 min of OGD. However, although this exposure is lethal to nearly all neurons, it is hours less than that needed to kill astrocytes. Astrocyte $\psi_{m}$ was rescued during OGD by cyclosporin A, a permeability transition pore blocker, and ${ }^{G} N$-nitro-arginine, a nitric oxide synthase inhibitor. Loss of mitochondrial membrane potential in astrocytes was not accompanied by depolarization of the plasma membrane. Recovery of astrocyte $\psi_{m}$ after reintroduction of $\mathrm{O}_{2}$ and glucose occurred over a surprisingly long period ( $>1 \mathrm{hr}$ ), suggesting that OGD caused specific, reversible changes in astrocyte mitochondrial physiology beyond the simple lack of $\mathrm{O}_{2}$ and glucose. Decreased $\psi_{m}$ was associated with a cyclosporin A-sensitive loss of cytochrome $c$ but not with activation of caspase-3 or caspase-9. Our data suggest that astrocyte mitochondrial depolarization could be a previously unrecognized event early in ischemia and that strategies that target the mitochondrial component of ischemic injury may benefit astrocytes as well as neurons.

Key words: tetramethylrhodamine ethyl ester; mitochondrial permeability transition pore; nitric oxide synthase; cyclosporin A; confocal microscopy; cortical cell cultures
Mitochondrial dysfunction is an early feature in nervous system ischemia. Functional studies on mitochondria isolated from ischemic brain (Sims et al., 1986; Sims, 1991) and metabolic imaging studies of brain during ischemia (Watanabe et al., 1994; Shiino et al., 1998; McCleary et al., 1999; Shadid et al., 1999) indicate that brief periods of ischemia result in transient mitochondrial respiratory defects that normalize rapidly after reperfusion (Schutz et al., 1973; Hillered et al., 1984; Sims et al., 1986). Longer periods of ischemia, however, result in a secondary, irreversible decline in mitochondrial function that occurs minutes to hours later (for review, see Siesjo et al., 1999). Although mitochondrial failure is associated with loss of mitochondrial membrane potential $\left(\psi_{m}\right)$ in many injury conditions (Green and Reed, 1998), it is not yet known whether impaired mitochondrial respiration during cerebral ischemia is accompanied by loss of $\psi_{m}$, because of the lack of sensitive and specific probes for $\psi_{m}$ in the intact brain. Suggestive evidence comes from reports by Fujimura et al. (1998), Andreyev et al. (1998), and Perez-Pinzon et al. (1999), who observed release of cytochrome c from mitochondria rapidly after reperfusion, and from a study by Krajewski et al. (1999), who observed release of caspase-9, an intramitochondrial caspase, after global ischemia.

Received April 10, 2001; revised May 31, 2001; accepted June 13, 2001.

This work was supported by National Institutes of Health Grant NS 32636 (L.L.D.).

S.A.R. and J.S.K.-H. contributed equally to this work.

Correspondence should be addressed to Dr. Laura L. Dugan, Department of Neurology, 660 South Euclid Avenue (Box 8111), Washington University School of Medicine, St. Louis, MO 63110. E-mail: duganl@neuro.wustl.edu.

Copyright (C) 2001 Society for Neuroscience 0270-6474/01/216608-09\$15.00/0
In addition, loss of $\psi_{m}$ has been directly demonstrated in hippocampal CA1 neurons exposed to oxygen-glucose deprivation (OGD) by the use of acute brain slice preparations (Bahar et al., 2000; Quick and Dugan, 2000).

The selective vulnerability of neurons to ischemic injury has been taken as an indication that neurons experience greater metabolic deterioration than do astrocytes, which are generally spared by the same ischemic insult. Furthermore, astrocytes contain glycogen stores that might be expected to buffer metabolic insults and help maintain mitochondrial function during ischemia. Extensive data indicate that astrocytes are critically involved in a number of processes that affect neuronal survival, such as glutamate uptake, maintenance of extracellular $\mathrm{pH}$ and potassium, $\mathrm{Ca}^{2+}$ buffering, and transfer of lactate and/or pyruvate to neurons as energy substrates (Magistretti et al., 1993; Forsyth, 1996; Vernadakis, 1996; Anderson and Swanson, 2000; Walz, 2000). A number of these astrocyte-support functions are dependent on mitochondrial membrane potential, and many of these same functions are impaired early in the ischemic period (Benveniste et al., 1984; Montgomery, 1994; Juurlink, 1997). This led us to speculate that deterioration of astrocyte mitochondrial function, specifically loss of $\psi_{m}$, might occur quite early in ischemia.

In the present study we found that astrocytes experience early and pronounced, but reversible, loss of mitochondrial membrane potential in response to oxygen-glucose deprivation, which does not result in astrocyte cell death. We characterized the time course and pharmacology of astrocyte mitochondrial depolarization and looked at specific biochemical events related to the loss 
of astrocyte $\psi_{m}$ induced by OGD. Our data suggest that decreased $\psi_{m}$ in astrocytes during ischemia could contribute to the cascade of events leading to ischemic cell death.

Parts of this paper have been published previously in abstract form (Reichert et al., 2000).

\section{MATERIALS AND METHODS}

Reagents. Tetramethylrhodamineethylester(TMRE),5,5',6,6'-tetrachloro1,1'3,3' 'tetraethylbenzimidazolocarbocyanine iodide (JC-1), rhodamine 123, bis-(1,3-dibutylbarbituric acid)trimethine oxonol [DiBAC4(3)], and propidium iodide (PI) were purchased from Molecular Probes (Eugene, OR). FK506 (tacrolimus) was purchased from Boehringer Mannheim (Indianapolis, IN), and cyclosporin A (CsA) was purchased from Sigma (St. Louis, MO) or Calbiochem (La Jolla, CA). MK-801 and 2,3-dihydroxy6-nitro-7-sulfamoyl-benzo(F)quinaoxaline (NBQX) were obtained from Research Biochemicals (Natick, MA), and ${ }^{\mathrm{G}} N$-nitro-arginine, oligomycin, carbonyl cyanide $p$-(trifluoromethoxy)phenylhydrazone (FCCP), and rotenone came from Sigma. Minimal Essential Medium without glutamine was purchased from Life Technologies (11430-022; Gaithersburg, MD). Other reagents were obtained from standard suppliers.

Cortical cell cultures. Cortical astrocyte cultures and mixed cortical neuron astrocyte cocultures were prepared as described previously (Dugan et al., 1995a). Astrocyte cultures were prepared from postnatal day 0 (P0) to P3 mouse pups by plating cortical cell suspensions on Primaria (Falcon) $75 \mathrm{~cm}^{2}$ flasks or $35 \mathrm{~mm}$ coverslip dishes (Mat-Tek) coated previously with poly-D-lysine and laminin. Mixed neuron-astrocyte cultures were prepared from fetal (embryonic day 15) Swiss-Webster mice (Charles River Laboratories, Wilmington, MA) by plating cortical cell suspensions onto a confluent bed of astrocytes. Cultures were treated with Ara-C $(10 \mu \mathrm{M}) 5-6 \mathrm{~d}$ after plating to inhibit proliferation of oligodendroglia and microglia. Cultures were fed biweekly with growth medium (Minimal Essential Medium plus $20 \mathrm{~mm}$ glucose, $26.2 \mathrm{~mm}$ $\mathrm{NaHCO}_{3}, 10 \%$ horse serum, and $2 \mathrm{mM}$ L-glutamine), until the final feeding at day 11 or 12 in vitro, when the medium was exchanged with Minimal Essential Medium supplemented with $2 \mathrm{~mm}$ L-glutamine. Astrocytes and mixed cultures were used for experiments $\sim 15 \mathrm{~d}$ after plating.

Oxygen-glucose deprivation. Combined oxygen-glucose deprivation was performed as described previously (Goldberg and Choi, 1993). Briefly, cells were placed in an anaerobic $\left(<0.02 \% \mathrm{O}_{2}\right.$ and $\left.5 \% \mathrm{CO}_{2}\right)$ environmental chamber (Forma Scientific), and the culture medium was exchanged with oxygenated, glucose-containing solution (for controls) or deoxygenated, glucose-free medium (for OGD groups). TMRE (100 nM) and all treatment drugs were included with the final medium exchange, i.e., at the onset of OGD. Control cultures were sealed immediately after the final exchange to retain $\mathrm{O}_{2}$ in the culture medium by placing a lid lined with Vaseline on the culture dish. OGD dishes were left unsealed. All cultures were then placed in the $37^{\circ} \mathrm{C}$ incubator within the anaerobic chamber for the duration of the experiment. OGD dishes were subsequently sealed with Vaseline at the end of the deprivation period. To study the role of nitric oxide synthase (NOS) in mitochondrial depolarization, cells were pretreated with ${ }^{\mathrm{G}} \mathrm{N}$-nitro-arginine $(1 \mathrm{~mm})$ for $4 \mathrm{hr}$, and then ${ }^{\mathrm{G}} N$-nitro-arginine $(1 \mathrm{~mm})$ was reapplied at the beginning of OGD. To determine the number of neurons and astrocytes killed by exposure to oxygen-glucose deprivation, propidium iodide $(50 \mu \mathrm{g} / \mathrm{ml}$ final concentration) was added to cultures $24 \mathrm{hr}$ after OGD, and the propidium iodide-positive astrocytes and neurons were counted using confocal microscopy to differentiate the cell layers.

Fluorescence imaging of mitochondrial membrane potential. To evaluate how TMRE fluorescence responded to changes in $\psi_{m}$ in cortical astrocytes, cultures loaded with TMRE $(100 \mathrm{nM})$ were exposed to mitochondrial-depolarizing agents including the complex I inhibitor rotenone $(30 \mu \mathrm{M})$, rotenone plus the $\mathrm{F}_{0} \mathrm{~F}_{1}$-ATPase inhibitor oligomycin $(2.5$ $\mu \mathrm{g} / \mathrm{ml})$, and the mitochondrial uncoupler FCCP (10 $\mu \mathrm{M})$. TMRE fluorescence [excitation $\lambda(\operatorname{Ex} \lambda)$ of $568 \mathrm{~nm}$ and emission $\lambda(\operatorname{Em} \lambda)>590 \mathrm{~nm}$ ] in mitochondria was visualized on a confocal microscope (Noran Odyssey) equipped with a krypton-argon laser (488 and $568 \mathrm{~nm}$ emission lines), using a $40 \times, 1.4$ numerical aperture Plan Apo oil-immersion objective (Nikon). To minimize photobleaching and other free radical dye reactions, laser intensity was minimized. To allow analysis of mitochondrial membrane potential in cultures during OGD, cultures were loaded with TMRE (100 nM) at the onset of OGD, culture dishes were then sealed with Vaseline before removal from the anaerobic chamber, and TMRE fluorescence in astrocyte mitochondria was assessed. Several fields per dish were selected at random by phase-contrast optics, and then fluorescence images were captured using MetaMorph imaging and analysis software. Fluorescence images were digitized at $640 \times 480$ pixels. Mitochondrial TMRE fluorescence was analyzed in images after background fluorescence was subtracted, using a mask function to eliminate nonmitochondrial pixels (determined as the background fluorescence in the cell nuclei). The average mitochondrial TMRE pixel intensity for each cell was then determined. JC-1 fluorescence was evaluated at Ex $\lambda$ of $488 \mathrm{~nm}$ and $\mathrm{Em} \lambda>525 \mathrm{~nm}$ (monomer) and $\mathrm{Ex} \lambda$ of $488 \mathrm{~nm}$ and $\mathrm{Em}$ $\lambda>590 \mathrm{~nm}$ (J-aggregates). Rhodamine 123 settings were Ex $\lambda$ of $488 \mathrm{~nm}$ and $\mathrm{Em} \lambda>515 \mathrm{~nm}$. Imaging protocols similar to that described for TMRE were used for these dyes. Astrocyte plasma membrane potential was imaged by confocal microscopy using DiBAC4(3) (1 $\mu \mathrm{M}$; excitation $\lambda=488 \mathrm{~nm}$; emission $\lambda=515 \mathrm{~nm}$ ) at the end of OGD.

Determination of cytochrome c release. Pure astrocyte cultures were exposed to OGD for 90-120 min until mitochondrial depolarization was observed in a sister culture used solely to monitor $\psi_{m}$. Astrocyte mitochondria were then isolated using a modification of the method described by Almeida and Medina (1997). Purity of the mitochondrial fraction was confirmed by assaying the enrichment in cytochrome oxidase activity (data not shown). Cells were washed with PBS, pH 7.4, harvested, and centrifuged at $700 \times g$ for $5 \mathrm{~min}$. The pellet was resuspended in isolation buffer ( $320 \mathrm{~mm}$ sucrose, $10 \mathrm{~mm}$ Tris-HCl, pH 7.2, and $1 \mathrm{~mm} \mathrm{EDTA-K}^{+}$) and homogenized. Homogenates were centrifuged at $1500 \times g$ for $5 \mathrm{~min}$ twice. Supernatants collected from both centrifugations were centrifuged at $17,000 \times g$ for $10 \mathrm{~min}$ at $4^{\circ} \mathrm{C}$. The pellet (mitochondrial fraction) was resuspended in $200-300 \mu \mathrm{l}$ of isolation buffer. The supernatant was further centrifuged at $200,000 \times g$ for $30 \mathrm{~min}$ at $4^{\circ} \mathrm{C}$ to remove membranes. Cytochrome $\mathrm{c}$ was quantified in the mitochondrial and cytoplasmic fractions using a Quantikine M cytochrome c ELISA kit from R \& D systems (Minneapolis, MN). A standard curve for cytochrome c was constructed using standards included in the kit. Because we expected relatively low concentrations of cytochrome $\mathrm{c}$ in the cytosol and wanted to increase our sensitivity for changes in cytosolic cytochrome c, the amount of protein analyzed for cytochrome c content in the cytosolic fractions was 2.5 times that of the mitochondrial fractions. The amount of protein per sample was determined using the BCA assay system (Pierce, Rockford, IL), and the concentration of cytochrome $\mathrm{c}$ was expressed as micrograms per milligram of protein.

Analysis of caspase-3 and caspase- 9 activity. To assay caspase- 3 and caspase-9 activity after OGD, astrocyte cultures grown in $100 \mathrm{~mm}$ dishes were exposed to $90-120$ min of OGD or control conditions and harvested; some cultures were exposed to OGD and then returned to medium containing $\mathrm{O}_{2}$ and glucose for $1 \mathrm{hr}$ before harvesting. Cells were washed twice with cold PBS, and after the final wash, $5 \mathrm{ml}$ of cold PBS was added to each dish, and the cells were scraped and transferred to a $15 \mathrm{ml}$ centrifuge tube for each sample. To rinse the dishes, $3 \mathrm{ml}$ of cold PBS was used and added to the $15 \mathrm{ml}$ tubes. Tubes were centrifuged at $2500 \mathrm{rpm}$ for $10 \mathrm{~min}$. The PBS was discarded, and the pellet was frozen at $-80^{\circ} \mathrm{C}$ until use. Caspase-3 activity was assayed using an EnzChek Caspase-3 Assay Kit (Molecular Probes) as per the assay kit instructions. The assay plate was incubated for $30 \mathrm{~min}$ at room temperature, and fluorescence from cleavage of the substrate (Z-DEVD-R110) was measured on a microplate fluorescent reader (FL600, Bio-Tek), using excitation $\lambda=490 \mathrm{~nm}$ and emission $\lambda=520 \mathrm{~nm}$.

Caspase-9 activity was determined using a mammalian ced-3 homolog 6/Caspase-9 Fluorometric Protease Assay Kit (Chemicon, Temecula, CA) as per the manufacturer's instructions. Cells were lysed in the lysis buffer included in the kit and then incubated on ice for $10 \mathrm{~min}$. Fifty microliters of each sample were added to a microtiter plate, followed by addition of $2 \times$ reaction buffer containing $10 \mathrm{~mm}$ DTT to each sample. Finally, Leu-Glu-(oMe)-His-Asp(oMe)-7-amino-4-trifluoromethyl coumarin substrate was added, and the plate was incubated at $37^{\circ} \mathrm{C}$ for $1-2$ $\mathrm{hr}$. Fluorescence was read on a fluorescence microplate reader, using emission $\lambda=400 \mathrm{~nm}$ and emission $\lambda=505 \mathrm{~nm}$. Caspase-appropriate inhibitors (included with each kit) were used to allow nonspecific cleavage of the fluorescent substrates to be subtracted.

\section{RESULTS}

Oxygen-glucose deprivation results in profound loss of astrocyte mitochondrial $\psi$

We used the potentiometric fluorescent compound TMRE to monitor astrocyte $\psi_{m}$. Both tetramethylrhodamine methyl ester (TMRM) and TMRE have been used to measure $\psi_{m}$ (Andreyev 


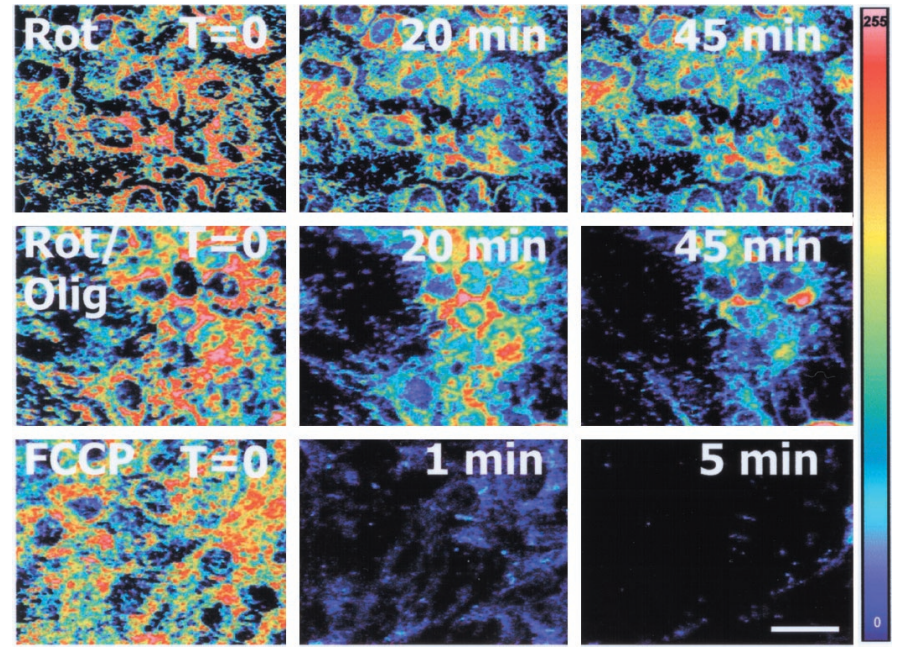

Figure 1. Mitochondrial depolarization results in a graded decline in TMRE fluorescence in astrocytes. Astrocytes were loaded with TMRE $(100 \mathrm{nM})$ and exposed to the mitochondrial depolarizing agents rotenone (complex I inhibitor), rotenone plus oligomycin $\left(\mathrm{F}_{0} \mathrm{~F}_{1}\right.$-ATPase inhibitor), and FCCP (mitochondrial protonophore). TMRE fluorescence, reflecting mitochondrial membrane potential, was visualized by confocal microscopy. TMRE fluorescence decreased after rapid depolarization by FCCP or gradual depolarization by rotenone or rotenone plus oligomycin. Panels are pseudocolor representations of TMRE fluorescence intensity (scale on right). Scale bar, $20 \mu \mathrm{m}$. Olig, Oligomycin; Rot, rotenone.

et al., 1998; Bindokas et al., 1998), but it has become clear that their fluorescence response to mitochondrial depolarization differs considerably depending on the concentration of the probe used and to some extent on the relationship of the plasma membrane and mitochondrial membrane potentials (Ward et al., 2000). We therefore set out to establish optimal conditions for the use of TMRE in cortical astrocytes. Using TMRE at $100 \mathrm{nM}$, we observed a reproducible, graded, and time-dependent decrease in fluorescence after mitochondrial depolarization produced by rotenone, rotenone plus oligomycin, or FCCP (Fig. 1). Concentrations of TMRE $>100 \mathrm{nM}$ demonstrated fluorescence unquenching and increased fluorescence with mitochondrial depolarization, and concentrations lower than $\sim 50 \mathrm{~nm}$ produced insufficient fluorescence to detect sensitively changes in $\psi_{m}$ without increasing laser intensity and subsequent phototoxicity. Although Ward et al. (2000) reported unquenchingmediated increases in fluorescence for TMRM at $100 \mathrm{nM}$, we failed to observe unquenching with this concentration of TMRE, possibly reflecting greater binding of TMRE to the mitochondrial matrix (Scaduto and Grotyohann, 1999) or unquenching that was more rapid than our earliest time point with FCCP (60 sec).

We then used TMRE to evaluate $\psi_{m}$ in cortical cultures exposed to OGD. Mixed cultures were exposed to OGD for periods ranging from 30 to $90 \mathrm{~min}$ in the presence of TMRE, and $\psi_{m}$ was assessed in neurons and the underlying astrocytes using confocal microscopy. Exposure to $30 \mathrm{~min}$ of OGD had no effect on astrocyte or neuronal $\psi_{m}$, but 40-60 min of OGD produced a progressive decline in astrocyte $\psi_{m}$, with near-complete loss of $\psi_{m}$ by $60 \mathrm{~min}$ of OGD (Fig. $2 A, B$ ). Neuronal mitochondria were also uniformly depolarized by $60 \mathrm{~min}$ of OGD but demonstrated a more variable loss of $\psi_{m}$ between 40 and 60 min of OGD, with depolarization generally, but not always, preceding the loss of $\psi_{m}$ in astrocytes (data not shown). Using $\operatorname{DiBAC}_{4}(3)$ to monitor astrocyte plasma membrane potential, we observed no change in plasma membrane potential after OGD (Fig. 3). Therefore, decreased TMRE fluorescence appears to reflect only changes in mitochondrial $\psi_{m}$ in these experiments.

We confirmed OGD-induced loss of $\psi_{m}$ with two other potentiometric dyes, JC-1 and rhodamine 123. Experiments with JC-1, a highly sensitive ratiometric probe for $\psi_{m}$, confirmed our observations with TMRE (data not shown). However, we found that JC-1 was less reliable than TMRE for our specific studies because, at the extremely low levels of $\psi_{m}$ that we observed, both JC-1 aggregates and monomers are lost from mitochondria, giving artifactually high fluorescence ratios. Similar observations were made with rhodamine 123 , but because of the prominent unquenching properties of this probe, we chose to use TMRE for subsequent experiments.

\section{Loss of astrocyte $\psi_{m}$ during OGD is blocked by inhibition of the mitochondrial permeability transition pore and NOS}

We next determined the pharmacology of astrocyte mitochondrial depolarization. Cyclosporin A, an inhibitor of both calcineurin and the mitochondrial membrane permeability transition pore (mPTP), partially prevented the loss of astrocyte $\psi_{m}$ (Table 1). However, FK506 (tacrolimus), which inhibits calcineurin but not the mPTP (Connern and Halestrap, 1994), failed to rescue $\psi_{m}$, suggesting that activation of the MPTP contributes to the OGD-induced loss of $\psi_{m}$. The general nitric oxide inhibitor ( ${ }^{\mathrm{G}} N$-nitro-arginine) also partially rescued astrocyte $\psi_{m}$. Protection by combined treatment with CsA and the NOS inhibitor was not significantly better than that with either agent alone. No effect of CsA or ${ }^{\mathrm{G}} N$-nitro-arginine on astrocyte $\psi_{m}$ under control conditions was observed (Table 1). Neither the NMDA receptor antagonist MK-801 nor the general AMPA/kainate receptor antagonist NBQX affected the mitochondrial depolarization induced by OGD.

\section{Recovery of astrocyte $\psi_{m}$ after reintroduction of oxygen and glucose after OGD is a delayed process}

If mitochondrial depolarization during OGD reflects the simple lack of glucose and oxygen, then readdition of the two metabolic substrates should allow rapid recovery of $\psi_{m}$. However, we found that when $\mathrm{O}_{2}$ and glucose were reintroduced to astrocytes after OGD, mitochondria required $>1 \mathrm{hr}$ to return to control levels of $\psi_{m}$ (Fig. 2C,D); mitochondrial membrane potential was still $<50 \%$ of control values $30 \mathrm{~min}$ after "reperfusion" (readdition of $\mathrm{O}_{2}$ and glucose) and was still only $85 \%$ of controls at $1 \mathrm{hr}$. However, mitochondrial membrane potential returned to control values after an additional hour in $\mathrm{O}_{2}$ and glucose.

We also noted that the morphology of repolarized mitochondria often appeared abnormal, even $1 \mathrm{hr}$ after readdition of $\mathrm{O}_{2}$ and glucose (Fig. 2E). Mitochondria were not obviously swollen, but there seemed to be a greater number of round "discrete" mitochondria with less filament-like structures and fewer intramitochondrial connections. These morphological changes also appeared to have resolved within 2-3 hr after reintroduction of $\mathrm{O}_{2}$ and glucose (Fig. 2E). Taken together, these data suggest that OGD causes persistent (i.e., $>1 \mathrm{hr}$ ) but reversible changes in astrocyte mitochondrial function and structure that are not attributable solely to lack of $\mathrm{O}_{2}$ and glucose. 

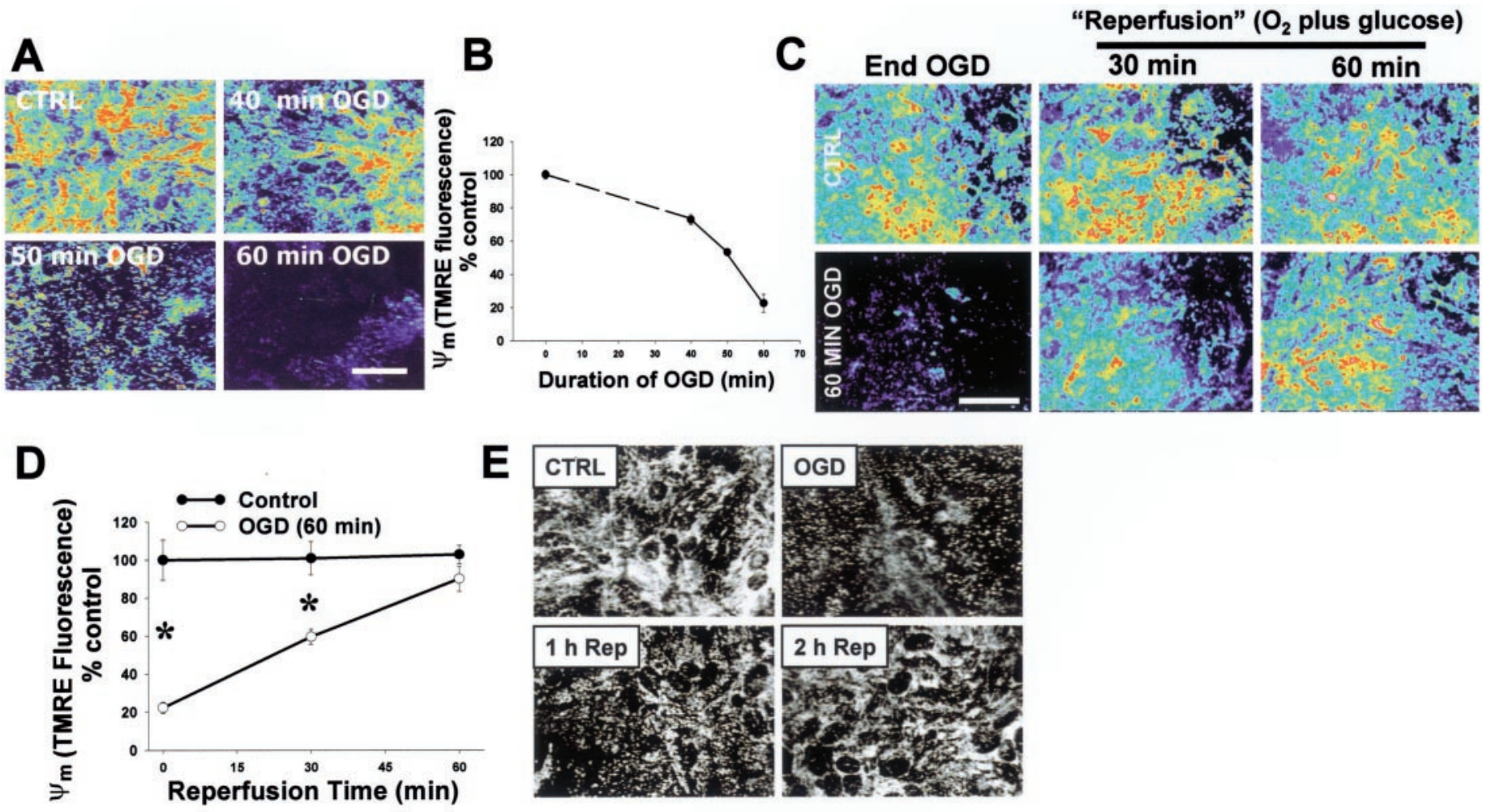

Figure 2. Mitochondrial membrane potential in cortical astrocytes exposed to OGD and subsequent reintroduction of oxygen and glucose. Mixed cortical cultures were exposed to OGD in the presence of the potentiometric dye TMRE. $A$, $C$, Mitochondrial $\psi$, as TMRE fluorescence, was evaluated in the astrocyte monolayer by confocal microscopy after 40,50, or $60 \mathrm{~min}$ of OGD $(A)$ or during recovery after reintroduction of oxygen and glucose after 60 min of OGD $(C)$. Control cultures were maintained in $21 \%$ oxygen and $5.5 \mathrm{~mm}$ glucose for $60 \mathrm{~min}$. $B$, $D$, The time course of mitochondrial TMRE fluorescence loss in astrocytes during OGD $(B)$ and the recovery of mitochondrial $\psi$ after readdition of oxygen and glucose $(D)$ are shown. Dashed line in $B$ indicates interpolation of TMRE fluorescence changes between 0 and 40 min. Values are mean TMRE fluorescence intensity (as $\%$ of control) \pm SEM; $n>100$ for all conditions from 3 or more independent experiments. ${ }^{*} p<0.05$ by ANOVA and Tukey's post hoc analysis. E, Astrocytes were exposed to OGD $(60 \mathrm{~min})$ or OGD followed by reintroduction of oxygen plus glucose for an additional 60 or 120 min. Alterations in mitochondrial morphology, visualized by TMRE fluorescence, were apparent after OGD, persisted after reintroduction of $\mathrm{O}_{2}$ plus glucose for 60 min, but had resolved by 120 min after addition of $\mathrm{O}_{2}$ plus glucose. Scale bar: $A, C, 20 \mu \mathrm{m}$. CTRL, Control; Rep, reperfusion.

\section{Cytochrome $\mathrm{c}$ is lost from mitochondria during astrocyte mitochondrial depolarization}

Loss of $\psi_{m}$ has been associated with release of cytochrome c from mitochondria during the course of apoptotic cell death in many cell types (Green and Reed, 1998). However, it is unclear whether mitochondrial depolarization will lead to release of cytochrome $\mathrm{c}$ in cells that are not destined to die. To determine whether prolonged, but nonlethal (Fig. 4), mitochondrial depolarization in astrocytes results in release of cytochrome c, we first confirmed that astrocytes cultured without neurons would undergo mitochondrial depolarization in response to OGD. We found that, although astrocytes grown in pure culture exhibited slower loss of $\psi_{m}$ than did astrocytes cocultured with neurons (Fig. 5), the ultimate magnitude of $\psi_{m}$ loss was similar whether astrocytes were cultured alone or with neurons. In addition, mitochondrial depolarization in pure astrocyte cultures was partially blocked by cyclosporin A (data not shown), mirroring our results in mixed cultures.

To determine whether prolonged mPTP-dependent loss of $\psi_{m}$ would lead to translocation of cytochrome $\mathrm{c}$ in astrocytes exposed to OGD (cells that are not in the process of dying), we measured cytochrome c content in the mitochondria and cytosol. Using a "sentinel" culture loaded with TMRE to monitor $\psi_{m}$, we then exposed pure astrocyte cultures to OGD until $\psi_{m}$ loss was observed. After mitochondrial depolarization was documented, astrocytes were harvested, and subcellular fractions were prepared.
OGD caused a significant decrease in mitochondrial cytochrome c content that was blocked by treatment with CsA (Fig. 6A). CsA did not significantly affect cytochrome c content in control cultures. Levels of cytochrome $\mathrm{c}$ in the cytosol at baseline were quite low, and no significant increase in cytosolic cytochrome c was observed after OGD (Fig. 6A). To determine whether cytochrome c loss from mitochondria continued to increase after reperfusion, cytochrome c was measured in astrocyte cultures that had been returned to normoxic glucose-containing medium for $1 \mathrm{hr}$ after exposure to OGD for $60 \mathrm{~min}$. We found that mitochondrial cytochrome $\mathrm{c}$ still trended toward lower values than did controls even $1 \mathrm{hr}$ after readdition of $\mathrm{O}_{2}$ and glucose but showed greater variability at this time point than at the end of OGD. Regardless, these data indicate that extensive further release of mitochondrial cytochrome $\mathrm{c}$ after reintroduction of $\mathrm{O}_{2}$ and glucose does not occur. To observe cytochrome c release in astrocytes undergoing apoptosis, astrocytes were treated with 0.2 $\mu \mathrm{M}$ staurosporine for $16 \mathrm{hr}$ (Fig. 6B). Cytochrome c levels in mitochondria in staurosporine-treated cells were lower than those in control astrocyte cultures.

\section{Caspase- 9 and caspase- 3 are not activated despite cytochrome c release in astrocytes after OGD}

Historically, release of cytochrome $\mathrm{c}$ from mitochondria has been associated with activation of caspase- 9 and caspase- 3 in in vitro, 


\section{A}
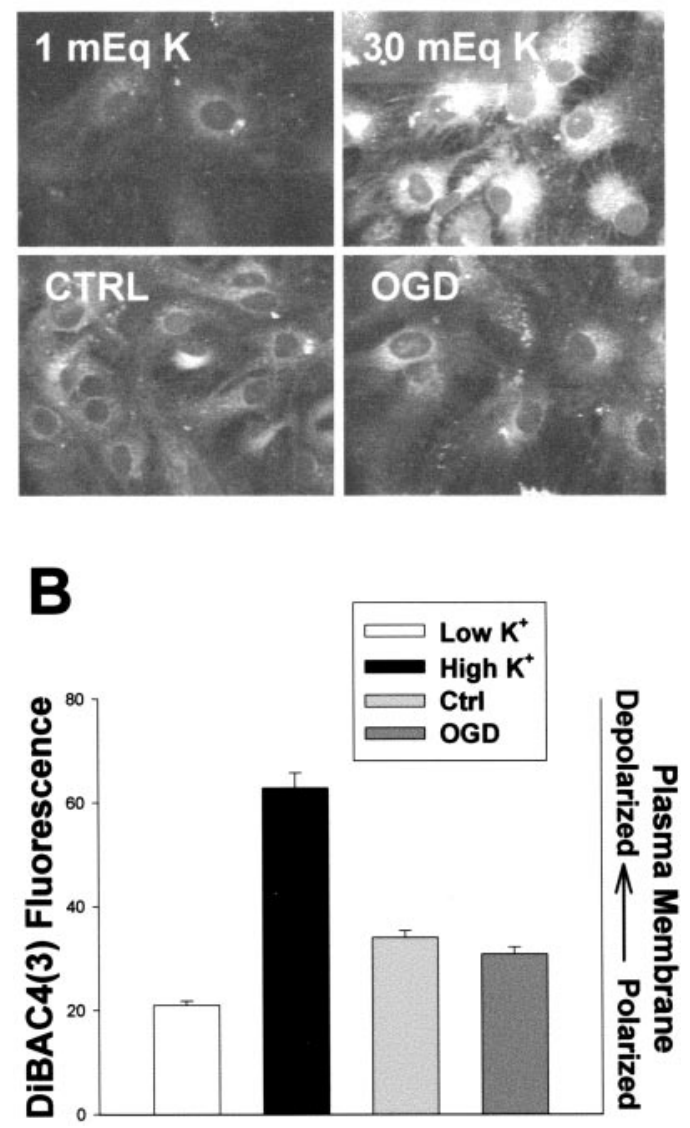

Figure 3. Plasma membrane potential in astrocytes exposed to low $\mathrm{K}^{+}(1$ $m E q \mathrm{~K})$, high $\mathrm{K}^{+}(30 \mathrm{mEq} \mathrm{K})$, control, and OGD conditions $(90-120$ $\mathrm{min})$ in the presence of DiBAC4(3) $(1 \mu \mathrm{M})$, a plasma membrane potentiometric dye. Control cultures were maintained in $21 \%$ oxygen and 5.5 mM glucose for $90-120 \mathrm{~min}$. $A$, DiBAC4(3) fluorescence was visualized by confocal microscopy (excitation $\lambda=488 \mathrm{~nm}$, emission $\lambda=515 \mathrm{~nm}$ ) at the end of OGD. $B$, There was no change in plasma membrane potential in astrocytes exposed to OGD compared with the control condition.

cell culture, and in vivo systems (for review, see Green and Reed, 1998). To determine whether loss of cytochrome $\mathrm{c}$ would activate caspase- 3 or caspase- 9 in cells not proceeding toward death, we assayed the activity of these two caspases in OGD-exposed astrocytes using fluorogenic substrates. No activation of either caspase was observed in astrocytes after exposure to OGD (Fig. 7). Activity levels in astrocytes exposed to OGD showed a nonsignificant $(p=0.09)$ trend toward lower values, whereas we easily detected increased caspase- 3 and caspase- 9 activity in astrocyte cultures induced to undergo apoptosis by treatment with staurosporine (Fig. 7). To determine whether caspase-3 activation might be delayed after cytochrome c was released during OGD, we measured caspase- 3 activity 1 and $2 \mathrm{hr}$ after reperfusion but found no increase in caspase- 3 activity at these later time points either (data not shown). Thus, although exposure to OGD results in release of cytochrome c from astrocyte mitochondria, activation of caspase-3 and caspase-9 is not initiated.

\section{DISCUSSION}

This paper describes three novel findings. First, astrocytes exposed to a relatively brief period of oxygen-glucose deprivation experience profound and prolonged mitochondrial depolariza-
Table 1. Pharmacology of OGD-induced loss of astrocyte mitochondrial $\Psi$ at the end of OGD

$\begin{array}{ll}\text { Condition } & \text { TMRE fluorescence } \\ \text { Mean } \pm \text { SEM }(n)\end{array}$

Cultures were exposed to OGD in the presence of the following drugs: the PTP inhibitor CsA, the calcineurin inhibitor FK506, the nitric oxide synthase inhibitor ${ }^{\mathrm{G}} N$-nitro-arginine ( $\left({ }^{\mathrm{G}} N\right.$-Arg), the NMDA antagonist MK-801 (MK), and the general AMPA/kainate receptor blocker NBQX. All treatments were initiated at the beginning of OGD, except ${ }^{\mathrm{G}} N$-Arg, which was applied $24 \mathrm{hr}$ before OGD (1 mM) and again at the beginning of OGD. CsA, but not FK506, partially reversed the loss of astrocyte $\Psi_{\mathrm{m}}$, suggesting a role for the PTP in $\Psi_{\mathrm{m}}$ loss. ${ }^{\mathrm{G}} N$-nitro-arginine also partially rescued astrocyte $\Psi_{\mathrm{m}}$, and the combination of CsA and ${ }^{\mathrm{G}} N$-Arg was slightly better than either drug alone. Values are mean TMRE fluorescence (\% control) \pm SEM. Groups were compared by ANOVA and Dunnett's post hoc analysis $(p<$ $0.01)$. glc, Glucose.

${ }^{a \mathrm{G}} N$-Arg $(1 \mathrm{~mm})$ was applied $4 \mathrm{hr}$ before OGD and then reapplied at the beginning of OGD.

${ }^{b}$ Significantly different from control.

${ }^{c}$ Significantly different from OGD.

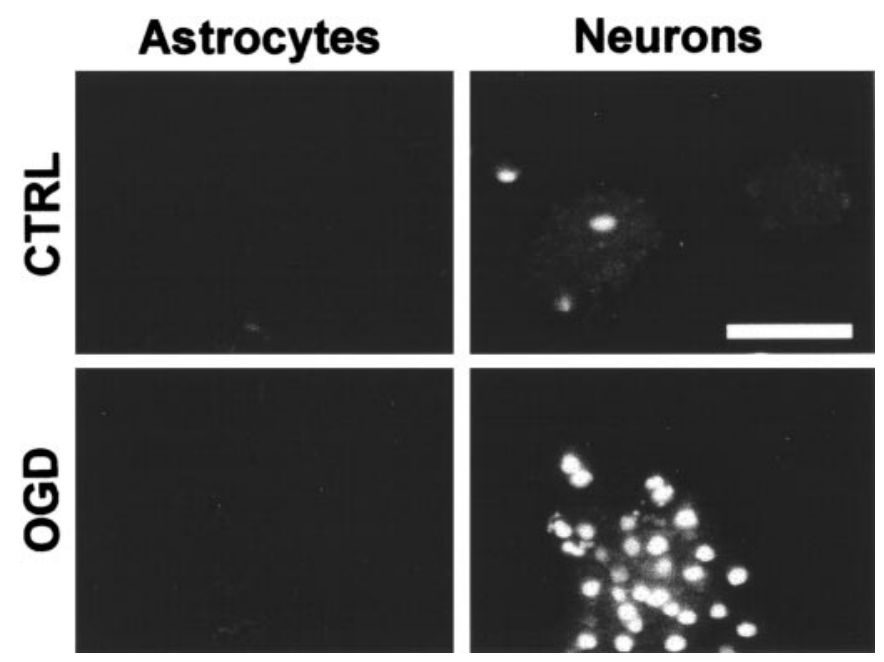

Figure 4. Determination of astrocyte and neuronal death after OGD in cortical cultures. Mixed cortical cultures were exposed to OGD or control conditions for $60 \mathrm{~min}$ and then returned to normoxic, glucose-containing solution for $24 \mathrm{hr}$ (data not shown) or $48 \mathrm{hr}$. The number of dead neurons and astrocytes was determined by staining with propidium iodide, followed by confocal microscopy to allow stained cells in the astrocyte layer (left) to be differentiated from the neurons above (right). PI-positive astrocytes per well in a 24-well culture plate were $35 \pm 12$ astrocytes in OGD-exposed cultures and $33 \pm 15$ astrocytes in control cultures (from 4 to 8 wells from 3 independent replications), both of which exhibit $<0.001 \%$ astrocyte death. Most neurons were dead $24 \mathrm{hr}$ after OGD. Scale bar, $50 \mu \mathrm{m}$.

tion that is not associated with subsequent cell death. Neurons appear to accelerate this process. To our knowledge, this is one of a handful of reports indicating that nontransformed cells can experience an extended duration of mitochondrial depolarization without overt injury or death. We also found that loss of astrocyte 

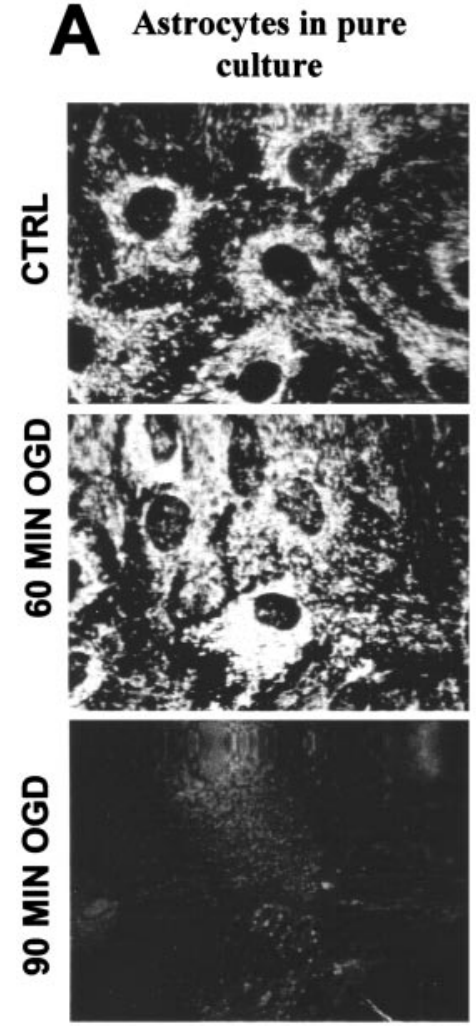

Astrocytes in mixed culture
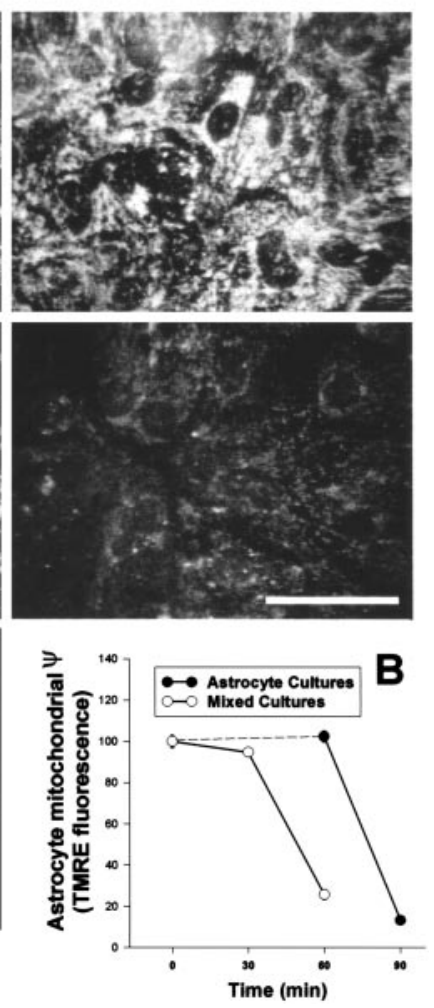

Figure 5. Comparison of OGD-induced mitochondrial depolarization in astrocytes cultured alone or cocultured with neurons. A, TMRE mitochondrial fluorescence was evaluated after $60 \mathrm{~min}$ of OGD in astrocytes cocultured with neurons (right) or after 60 and $90 \mathrm{~min}$ of OGD in astrocytes grown alone (left $)$. The optical sectioning ability of the confocal allowed astrocyte mitochondrial fluorescence to be differentiated from that of the overlying neurons in mixed cultures. Astrocytes cultured alone showed delayed loss of $\psi_{m}$ compared with astrocytes cultured with neurons. Scale bar, $20 \mu \mathrm{m}$. $B$, Quantitative analyses of mitochondrial TMRE fluorescence in the two culture types are shown. Values are mean TMRE fluorescence intensity (\% of control) \pm SEM; $n>50$ cells from 3 independent experiments. In $B$, the dashed line represents interpolation of TMRE fluorescence in astrocytes between 0 and $60 \mathrm{~min}$.

$\psi_{m}$ during OGD was mediated, in part, by opening of the MPTP and by NOS activity, providing the first direct evidence that the mPTP may contribute to astrocyte mitochondrial dysfunction under conditions of energy deprivation, suggesting that neuroprotection observed with CsA in CNS ischemia might reflect actions on astrocytes as well as neurons. Finally, we demonstrate that mitochondrial depolarization is accompanied by loss of cytochrome $\mathrm{c}$ from mitochondria without subsequent activation of caspase- 3 and caspase-9. These data support the idea that astrocyte mitochondrial dysfunction may be an early feature of ischemia, despite the relative invulnerability of astrocytes to ischemic injury.

Using OGD in cortical cell cultures to model many of the metabolic aspects of ischemia, we found that astrocytes and neurons experience early mitochondrial depolarization, beginning 45 min after the onset of OGD and progressing to nearcomplete depolarization by $60 \mathrm{~min}$ of OGD. This $15-20 \mathrm{~min}$ period corresponds to the time window during which extracellular glutamate levels begin to rise and irreversible neuronal injury is initiated in this model (Goldberg and Choi, 1993; Bruno et al.,
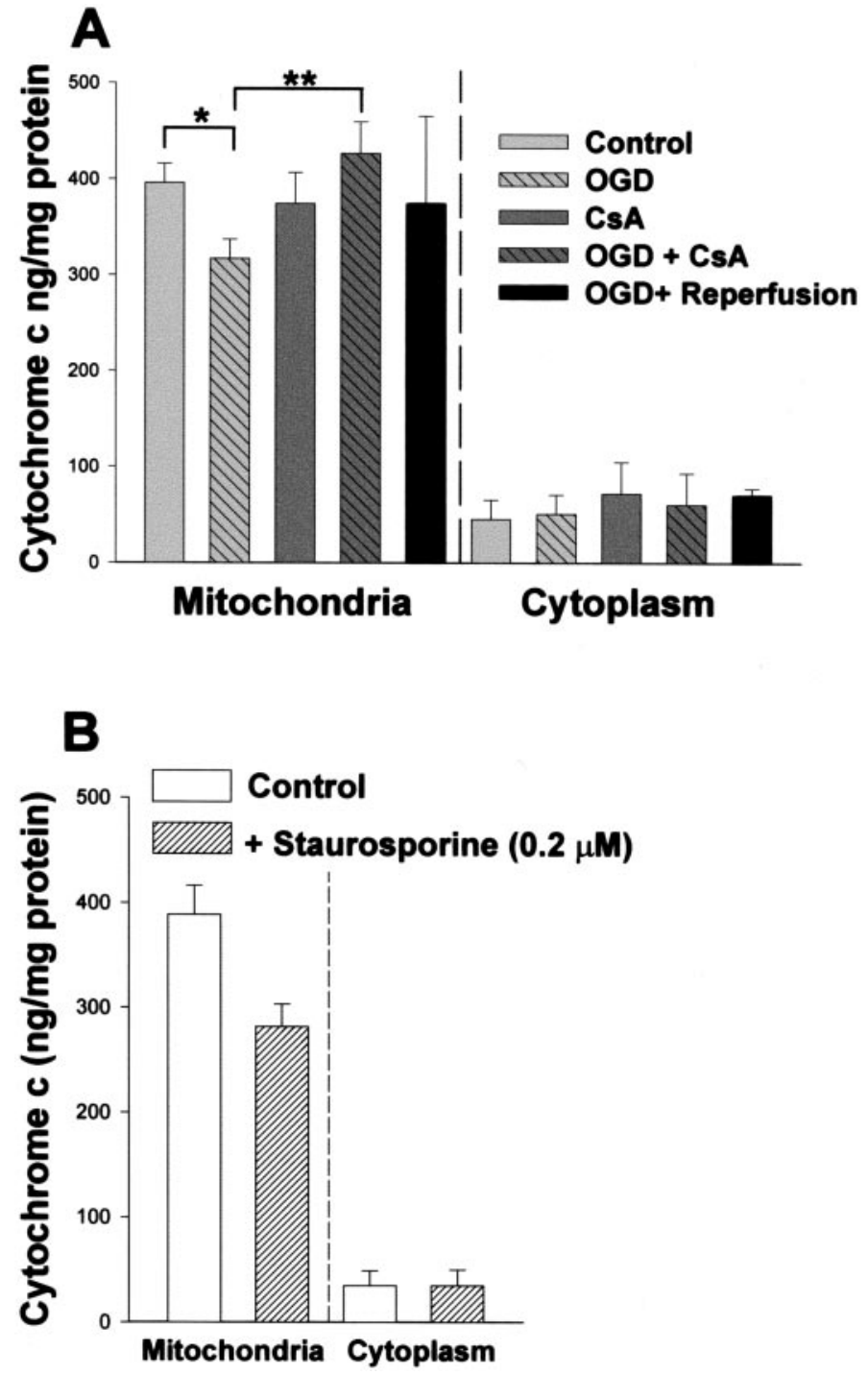

Figure 6. Cytochrome c loss from depolarized astrocyte mitochondria after OGD. Astrocytes were exposed to $90-120 \mathrm{~min}$ of OGD. A, After mitochondrial depolarization was observed, mitochondrial and cytosolic fractions were prepared and assayed for cytochrome c concentration. The left half of the graph shows cytochrome c concentration (per milligram of protein) in the mitochondrial fraction. The right half shows cytochrome $\mathrm{c}$ concentration in the cytosol. Values are the mean $\pm \mathrm{SEM} ; n=4$ independent replications for control and OGD only; 3 additional replications included CsA conditions $\left(^{*} p<0.05\right.$, significantly different from control, and ${ }^{* *} p<0.05$, significantly different from OGD, by ANOVA and Tukey's post hoc analysis). B, Apoptosis in astrocyte cultures was initiated by staurosporine at $0.2 \mu \mathrm{M}$. Cytochrome c was measured as nanograms per milligram of protein as stated above. Mitochondrial cytochrome c levels are lower in staurosporine-treated cells.

1994). However, injury to cortical neurons exposed to OGD is excitotoxic and mediated via NMDA receptors (Goldberg and Choi, 1993), and NMDA receptor activation can cause loss of $\psi_{m}$ in the absence of OGD (Dugan et al., 1995b; Nieminen et al., 1996; Schinder et al., 1996; White and Reynolds, 1996). Mitochondrial depolarization in neurons during OGD may simply reflect this excitotoxic component of injury. In contrast, astrocytes are not injured by this duration of OGD; astrocyte death is not initiated until OGD is extended to $4 \mathrm{hr}$ (Goldberg and Choi, 1993).

Mitochondrial depolarization in astrocytes precedes substan- 

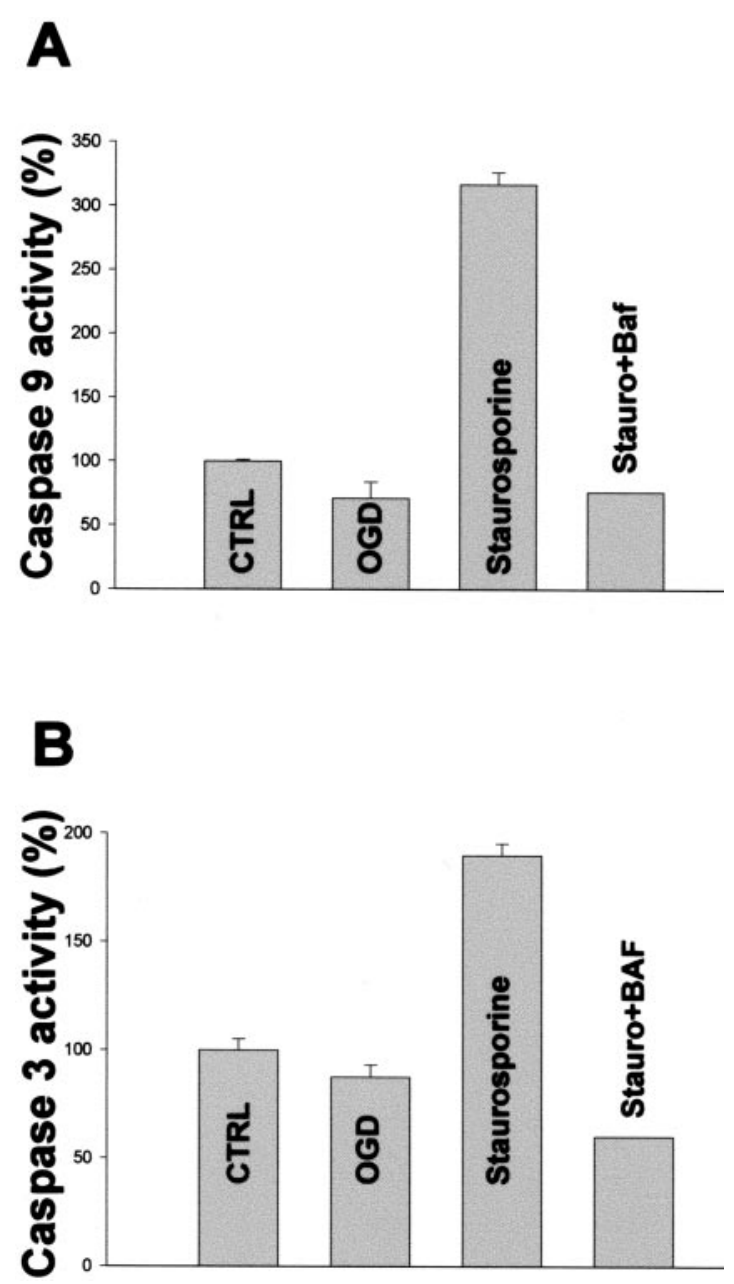

Figure 7. Activity of caspase- 3 and caspase- 9 in astrocytes exposed to OGD. Astrocytes were exposed to 90-120 min of OGD until mitochondrial depolarization was confirmed, and then astrocytes were harvested and assayed for caspase-9 $(A)$ or caspase-3 $(B)$ activity (\% control) using fluorogenic substrates. No activation of either caspase was observed in astrocytes exposed to OGD. In contrast, caspase-3 and caspase- 9 activation was easily observed in astrocytes undergoing apoptosis initiated by staurosporine at $0.2 \mu \mathrm{M}$. Values are the mean \pm SEM. Error bars for astrocytes exposed to staurosporine and Boc-Asp $(\mathrm{oMe})-\mathrm{CH}_{2} \mathrm{~F}(B A F)$ in $A$ and $B$ are not visible. Stauro, staurosporine.

tial loss of ATP. As reported previously, ATP levels were still $70 \%$ of control levels in cortical cultures exposed to $60 \mathrm{~min}$ of OGD (Bruno et al., 1994). Most of this ATP loss was likely to reflect energy depletion in neurons, rather than astrocytes, because neurons experienced collapse of their plasma membrane potential by $60 \mathrm{~min}$ of OGD, whereas astrocytes maintain their plasma membrane potential and tolerate an additional 2-3 hr of OGD without injury. Moreover, in the absence of OGD, astrocytes are clearly capable of using ATP to maintain $\psi_{m}$, running the $\mathrm{F}_{0} \mathrm{~F}_{1}$-ATPase "in reverse" to support $\psi_{m}$. Inhibition of electron entry via complex I by rotenone caused only a minimal decrease in astrocyte $\psi_{m}$ (Fig. 1), whereas coapplication of rotenone and the $\mathrm{F}_{0} \mathrm{~F}_{1}$-ATPase inhibitor oligomycin resulted in pronounced mitochondrial depolarization. Why astrocytes fail to use this compensatory mechanism during OGD is unclear, but taken together, these data raise the question of why astrocytes experience such early mitochondrial depolarization during OGD and whether neurons might, in fact, signal to astrocytes to initiate mitochondrial depolarization. This idea is supported by a growing body of literature describing extensive communication between neurons and astrocytes (Kimelberg and Norenberg, 1989; Magistretti et al., 1993; Giaume and McCarthy, 1996) and by our observation that astrocytes exposed to OGD in the absence of neurons required nearly twice as long to experience loss of $\psi_{m}$. Further studies will explore the possibility that communication between neurons and astrocytes is involved in early changes in astrocyte mitochondrial function during OGD.

Loss of astrocyte $\psi_{m}$ during OGD involved the mPTP, a voltage-gated channel that allows molecules and ions with a mass of $<1500$ Da to pass through the mitochondrial membrane. Activation of the mPTP can lead to mitochondrial depolarization and in certain cell types to release of cytochrome c from mitochondria (Halestrap et al., 2000). Assembly of the mPTP is triggered by several stimuli, including fatty acids, accumulation of mitochondrial calcium, and oxidative stress, events that occur during ischemia-reperfusion injury. In astrocytes exposed to OGD, $\psi_{m}$ was partly rescued by cyclosporin A. Inhibition of NOS also helped to maintain $\psi_{m}$ during OGD, implicating NO/peroxynitrite in astrocyte mitochondrial depolarization. Cortical astrocytes have little inducible NOS (iNOS) expression at baseline, although they can be stimulated to express iNOS by cytokines (Hewett et al., 1994). Mitochondrial NOS (Giulivi et al., 1998) in astrocytes has not been definitively established because of the lack of specific probes for this nitric oxide-generating system. Both nitric oxide and peroxynitrite can inhibit mitochondrial metabolism and respiration at a number of points in the metabolic machinery (Brown and Borutaite, 1999). However, the combination of ${ }^{\mathrm{G}} \mathrm{N}$-nitro-arginine and CsA was not significantly more effective than either agent alone, suggesting that peroxynitrite may be acting via opening of the mPTP to cause astrocyte mitochondrial depolarization (Scarlett et al., 1996). Additional processes, such as activation of mitochondrial-uncoupling proteins, may contribute to mitochondrial depolarization. Involvement of glutamate receptor-mediated calcium entry and direct uncoupling by $\mathrm{Ca}^{2+}$ are unlikely because the AMPA/kainate receptor antagonist NBQX had no effect on $\psi_{m}$ loss.

Numerous studies have shown that mitochondrial depolarization is linked to translocation of cytochrome $\mathrm{c}$ from mitochondria to the cytosol (see Green and Reed, 1998). However, this association is not universal (Bossy-Wetzel et al., 1998; Krohn et al., 1999) and has been studied primarily in cells undergoing apoptotic death. Activation of the mPTP can also initiate release of cytochrome c from mitochondria (Halestrap et al., 2000). We observed a $20 \%$ loss of cytochrome c from mitochondria at the end of OGD that was partly blocked by CsA, suggesting involvement of the mPTP. Release of cytochrome $c$ was not accompanied by activation of either caspase- 9 or caspase-3, suggesting that cytochrome c was blocked from activating procaspase-9. Surprisingly, loss of cytochrome $\mathrm{c}$ from mitochondria was not accompanied by significant accumulation in the cytoplasm. We speculate that this reflects rapid modification and/or degradation of cytochrome c after its release, as has been proposed previously (Neame et al., 1998; Putcha et al., 2000). In the latter study, release of cytochrome c was sufficient to activate caspase-3 but did not result in a detectable increase in cytoplasmic cytochrome $\mathrm{c}$ at any time point. The mechanism(s) by which cytochrome $\mathrm{c}$ is cleared from the cytosol after release from mitochondria remains to be determined. Studies on purified cytochrome c have shown that the oxidation state of the cytochrome (Wang and Kallenbach, 1998) and the interaction of the protein with anionic lipids 
(deJhong et al., 1995) can enhance its susceptibility to proteolysis, but whether either of these factors contributes to removal of cytochrome c from the cytoplasm will need to be established. Regardless, determining how cells terminate such potentially proapoptotic signals is likely to be an important area for future study.

Loss of mitochondrial $\psi_{m}$ during OGD produced persistent but reversible changes in astrocyte mitochondrial structure and function. Although $\psi_{m}$ eventually recovered to control levels, normalization required more than an hour, suggesting persistent inhibition of the electron transport chain or loss of critical metabolic intermediates, such as adenine nucleotides (Sims, 1991). Ultrastructural changes in mitochondria including swelling and a decrease in the syncytial nature of astrocyte mitochondria were observed after OGD plus reperfusion and required $\sim 2 \mathrm{hr}$ to reverse. These ultrastructural changes after OGD plus reperfusion may correspond to the mitochondrial swelling and matrix alterations reported in early postischemic brain by electron microscopy (Petito and Babiak, 1982), suggesting that derangements in mitochondrial function during ischemia may occur in both astrocytes and neurons.

Depolarization of astrocyte mitochondria during OGD might have both beneficial and harmful effects on neuronal survival. Short-term loss of $\psi_{m}$ would allow astrocytes to shift use of glycogen and glucose temporarily away from aerobic metabolism to glycolysis, increasing the amount of lactate available for delivery to metabolically impaired neurons. This might be tolerated for as long as astrocyte glycogen stores were available. However, prolonged loss of $\psi_{m}$ in astrocytes might be expected to have injury-promoting effects during CNS ischemia. Astrocytes are involved in the normal maintenance of brain homeostasis, including several energy-dependent functions necessary for normal neuronal activity, e.g., regulation of extracellular $\mathrm{K}^{+}, \mathrm{pH}$, and osmolality, export of metabolic intermediates, and rapid uptake of neurotransmitters (Kimelberg and Norenberg, 1989; Magistretti et al., 1993; Walz, 2000). The ability of astrocytes to maintain these functions may, in fact, be a critical determinant of neuronal survival after ischemia (Juurlink, 1997; Stanimirovic et al., 1997; Aschner et al., 1999; Marrif and Juurlink, 1999). Loss of $\psi_{m}$ and eventual energy failure in astrocytes might lead to an inability to provide these critical support functions during ischemia, thus exacerbating ischemic injury to neurons.

In summary, our data suggest that astrocyte mitochondrial dysfunction may be a relatively unexplored but important early step in the cascade of events involved in ischemic injury. In light of recent studies showing neuroprotection by $\mathrm{CsA}$ in in vivo models of ischemia (Siesjo et al., 1999), our data indicate that astrocytes, in addition to neurons, might be therapeutic targets of CsA neuroprotection. In addition, in agreement with studies suggesting that astrocytes may be as vulnerable as neurons to ischemic injury in certain brain regions, we speculate that astrocyte mitochondrial depolarization might contribute to astrocyte death during ischemia. Finally, astrocytes exposed to OGD may provide a model system in which to study aspects of mitochondrial dysfunction that are not specifically involved in cell death.

\section{REFERENCES}

Almeida A, Medina JM (1997) Isolation and characterization of tightly coupled mitochondria from neurons and astrocytes in primary culture. Brain Res 764:167-172.

Anderson CM, Swanson RA (2000) Astrocyte glutamate transport: review of properties, regulation, and physiological functions. Glia $32: 1-14$.
Andreyev AY, Fahy B, Fiskum G (1998) Cytochrome c release from brain mitochondria is independent of the mitochondrial permeability transition. FEBS Lett 439:373-376.

Aschner M, Allen JW, Kimelberg HK, LoPachin RM, Streit WJ (1999) Glial cells in neurotoxicity development. Annu Rev Pharmacol Toxicol 39:151-173

Bahar S, Fayuk D, Somjen GG, Aitken PG, Turner DA (2000) Mitochondrial and intrinsic optical signals imaged during hypoxia and spreading depression in rat hippocampal slices. J Neurophysiol 84:311-324.

Benveniste H, Drejer J, Schousboe A, Diemer NH (1984) Elevation of the extracellular concentrations of glutamate and aspartate in rat hippocampus during transient cerebral ischemia monitored by intracerebral microdialysis. J Neurochem 43:1369-1374.

Bindokas VP, Lee CC, Colmers WF, Miller RJ (1998) Changes in mitochondrial function resulting from synaptic activity in the rat hippocampal slice. J Neurosci 18:4570-4587.

Bossy-Wetzel E, Newmeyer DD, Green DR (1998) Mitochondrial cytochrome c release in apoptosis occurs upstream of DEVD-specific caspase activation and independently of mitochondrial transmembrane depolarization. EMBO J 17:37-49.

Brown GC, Borutaite V (1999) Nitric oxide, cytochrome c and mitochondria. Biochem Soc Symp 66:17-25.

Bruno VMG, Goldberg MP, Dugan LL, Giffard RG, Choi DW (1994) The effect of hypothermia on oxygen-glucose deprivation and excitatory amino acid induced injury in cortical cultures. J Neurochem 63:1398-1406.

Connern CP, Halestrap AP (1994) Recruitment of mitochondrial cyclophilin to the mitochondrial inner membrane under conditions of oxidative stress that enhance the opening of a calcium-sensitive nonspecific channel. Biochem J 302:321-324.

deJhong HH, Ritsema T, Killian JA (1995) Lipid specificity for membrane mediated partial unfolding of cytochrome c. FEBS Lett 360:255-260.

Dugan LL, Bruno VMG, Amagasu SM, Giffard RG (1995a) Glia modulate the response of murine cortical neurons to excitotoxicity: glia exacerbate AMPA neurotoxicity. J Neurosci 15:4545-4555.

Dugan LL, Sensi SL, Canzoniero LMT, Handran SD, Rothman SM, Lin T-S, Goldberg MP, Choi DW (1995b) Mitochondrial production of reactive oxygen species in cortical neurons following exposure to $N$-methyl-D-aspartate. J Neurosci 15:6377-6388.

Forsyth RJ (1996) Astrocytes and the delivery of glucose from plasma to neurons. Neurochem Int 28:231-241.

Fujimura M, Morita-Fujimura Y, Murakami K, Kawase M, Chan PH (1998) Cytosolic redistribution of cytochrome c after transient focal cerebral ischemia in rats. J Cereb Blood Flow Metab 18:1239-1247.

Giaume C, McCarthy KD (1996) Control of gap-junctional communication in astrocytic networks. Trends Neurosci 19:319-325.

Giulivi C, Poderoso JJ, Boveris A (1998) Production of nitric oxide by mitochondria. J Biol Chem 273:11038-11043.

Goldberg MP, Choi DW (1993) Combined oxygen and glucose deprivation in cortical culture: calcium-dependent and calcium-independent mechanisms of neuronal injury. J Neurosci 13:3510-3524.

Green DR, Reed JC (1998) Mitochondria and apoptosis. Science 281:1309-1312.

Halestrap AP, Doran E, Gillespie JP, O’Toole A (2000) Mitochondria and cell death. Biochem Soc Trans 28:170-177.

Hewett SJ, Csernansky CA, Choi DW (1994) Selective potentiation of NMDA-induced neuronal injury following induction of astrocytic iNOS. Neuron 13:487-494.

Hillered L, Siesjo BK, Arfors KE (1984) Mitochondrial response to transient forebrain ischemia and recirculation in the rat. $\mathrm{J}$ Cereb Blood Flow Metab 4:438-446.

Juurlink BH (1997) Response of glial cells to ischemia: roles of reactive oxygen species and glutathione. Neurosci Biobehav Rev 21:151-166.

Kimelberg HK, Norenberg MD (1989) Astrocytes. Sci Am 260:66-72, 74, 76.

Krajewski S, Krajewska M, Ellerby LM, Welsh K, Xie Z, Deveraux QL, Salvesen GS, Bredesen DE, Rosenthal RE, Fiskum G, Reed JC (1999) Release of caspase-9 from mitochondria during neuronal apoptosis and cerebral ischemia. Proc Natl Acad Sci USA 96:5752-5757.

Krohn AJ, Wahlbrink T, Prehn JH (1999) Mitochondrial depolarization is not required for neuronal apoptosis. J Neurosci 19:7394-7404.

Magistretti PJ, Sorg O, Yu N, Martin JL, Pellerin L (1993) Neurotransmitters regulate energy metabolism in astrocytes: implications for the metabolic trafficking between neural cells. Dev Neurosci 15:306-312.

Marrif H, Juurlink BH (1999) Astrocytes respond to hypoxia by increasing glycolytic capacity. J Neurosci Res 57:255-260.

McCleary AJ, Gower S, McGoldrick JP, Berridge J, Gough MJ (1999) Does hypothermia prevent cerebral ischaemia during cardiopulmonary bypass? Cardiovasc Surg 7:425-431.

Montgomery DL (1994) Astrocytes: form, functions, and roles in disease. Vet Pathol 31:145-167.

Neame SJ, Rubin LL, Philpott KL (1998) Blocking cytochrome c activity within intact neurons inhibits apoptosis. J Cell Biol 142:1583-1593. 
Nieminen AL, Petrie TG, Lemasters JJ, Selman WR (1996) Cyclosporin A delays mitochondrial depolarization induced by $N$-methyl-Daspartate in cortical neurons: evidence of the mitochondrial permeability transition. Neuroscience 75:993-997.

Perez-Pinzon MA, Xu GP, Born J, Lorenzo J, Busto R, Rosenthal M, Sick TJ (1999) Cytochrome C is released from mitochondria into the cytosol after cerebral anoxia or ischemia. J Cereb Blood Flow Metab 19:39-43.

Petito CK, Babiak T (1982) Early proliferative changes in astrocytes in postischemic noninfarcted rat brain. Ann Neurol 11:510-518.

Putcha GV, Deshmukh M, Johnson Jr EM (2000) Inhibition of apoptotic signaling cascades causes loss of trophic factor dependence during neuronal maturation. J Cell Biol 149:1011-1018.

Quick KL, Dugan LL (2000) Characterization of mitochondrial membrane potential alterations in an acute slice model of hypoxia-ischemia. J Neurotrauma 17:941.

Reichert SA, Kim-Han JS, Dugan LL (2000) Astrocytes tolerate prolonged loss of mitochondrial membrane potential induced by oxygenglucose deprivation. J Neurotrauma 17:962.

Scaduto Jr RC, Grotyohann LW (1999) Measurement of mitochondrial membrane potential using fluorescent rhodamine derivatives. Biophys $\mathbf{J}$ 76:469-477.

Scarlett JL, Packer MA, Porteous CM, Murphy MP (1996) Alterations to glutathione and nicotinamide nucleotides during the mitochondrial permeability transition induced by peroxynitrite. Biochem Pharmacol 52:1047-1055

Schinder AF, Olson EC, Spitzer NC, Montal M (1996) Mitochondrial dysfunction is a primary event in glutamate neurotoxicity. J Neurosci 16:6125-6133.

Schutz H, Silverstein PR, Vapalahti M, Bruce DA, Mela L, Langfitt TW (1973) Brain mitochondrial function after ischemia and hypoxia. II. Normotensive systemic hypoxemia. Arch Neurol 29:417-419.

Shadid M, Hiltermann L, Monteiro L, Fontijn J, Van Bel F (1999) Near infrared spectroscopy-measured changes in cerebral blood volume and cytochrome aa3 in newborn lambs exposed to hypoxia and hypercapnia, and ischemia: a comparison with changes in brain perfusion and $\mathrm{O}_{2}$ metabolism. Early Hum Dev 55:169-182.

Shiino A, Matsuda M, Handa J, Chance B (1998) Poor recovery of mitochondrial redox state in CA1 after transient forebrain ischemia in gerbils. Stroke 29:2421-2424.

Siesjo BK, Elmer E, Janelidze S, Keep M, Kristian T, Ouyang YB Uchino H (1999) Role and mechanisms of secondary mitochondrial failure. Acta Neurochir Suppl (Wien) 73:7-13.

Sims NR (1991) Selective impairment of respiration in mitochondria isolated from brain subregions following transient forebrain ischemia in the rat. J Neurochem 56:1836-1844.

Sims NR, Finegan JM, Blass JP (1986) Effects of postdecapitative ischemia on mitochondrial respiration in brain tissue homogenates. J Neurochem 47:506-511.

Stanimirovic DB, Ball R, Durkin JP (1997) Glutamate uptake and Na,KATPase activity in rat astrocyte cultures exposed to ischemia. Acta Neurochir Suppl (Wien) 70:1-3.

Vernadakis A (1996) Glia-neuron intercommunications and synaptic plasticity. Prog Neurobiol 49:185-214.

Walz W (2000) Role of astrocytes in the clearance of excess extracellular potassium. Neurochem Int 36:291-300.

Wang L, Kallenbach NR (1998) Proteolysis as a measure of the free energy difference between cytochrome $\mathrm{c}$ and its derivatives. Protein Sci 7:2460-2464.

Ward MW, Rego AC, Frenguelli BG, Nicholls DG (2000) Mitochondrial membrane potential and glutamate excitotoxicity in cultured cerebellar granule cells. J Neurosci 20:7208-7219.

Watanabe M, Harada N, Kosaka H, Shiga T (1994) Intravital microreflectometry of individual pial vessels and capillary region of rat. J Cereb Blood Flow Metab 14:75-84.

White RJ, Reynolds IJ (1996) Mitochondrial depolarization in glutamate-stimulated neurons: an early signal specific to excitotoxin exposure. J Neurosci 16:5688-5697. 